Supporting Information

\title{
Regulatory Switching by Concerted Motions on the Microsecond Timescale of the Oxygen Sensor Protein FixL
}

\author{
Takeo Yamawaki, ${ }^{\dagger}$ Misao Mizuno, ${ }^{\dagger}$ Haruto Ishikawa, ${ }^{\dagger}$ Kazuhiro Takemura," \\ Akio Kitao, " Yoshitsugu Shiro, ${ }^{\ddagger}$ and Yasuhisa Mizutani ${ }^{\star *}$ \\ tDepartment of Chemistry, Graduate School of Science, Osaka University, 1-1 \\ Machikaneyama, Toyonaka, Osaka 560-0043, Japan.
}

ISchool of Life Science and Technology, Tokyo Institute of Technology, 2-12-1 Ookayama, Meguro, Tokyo 152-8550, Japan.

Graduate School of Life Science, University of Hyogo, 3-2-1 Kouto, Kamigori, Ako, Hyogo 678-1297, Japan. 


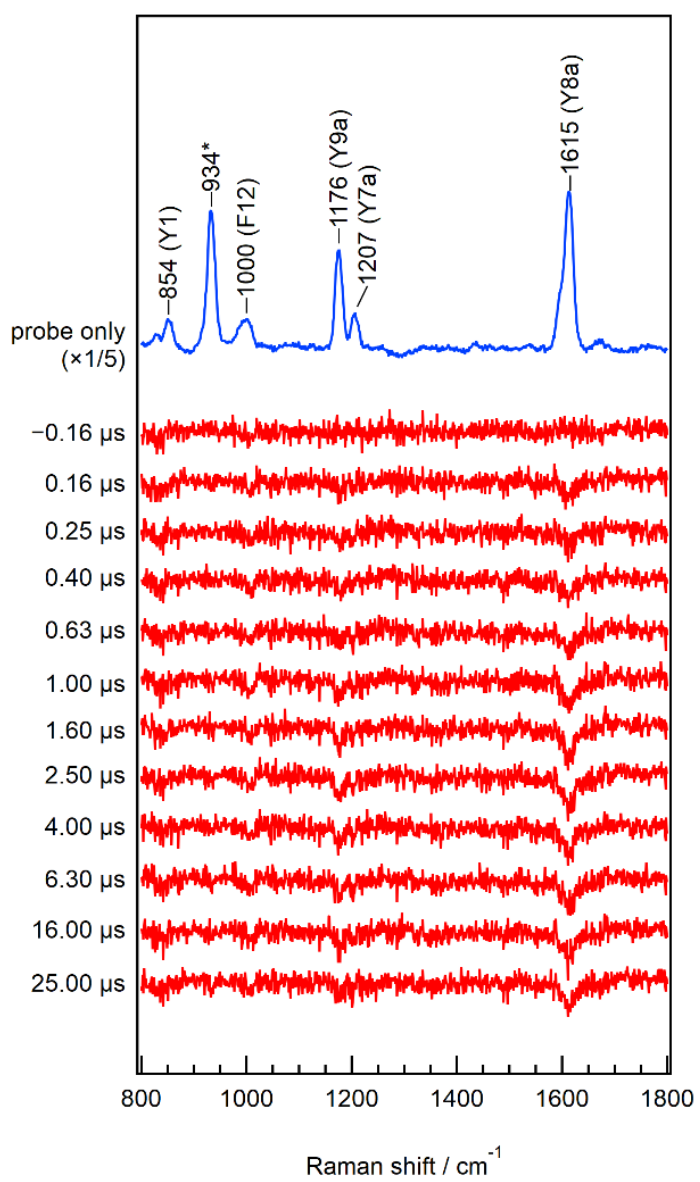

Figure S1. Time-resolved UVRR spectra at the indicated delay times following photolysis of the carbonmonoxy form of WT SmFixL. The top trace shows the probe-only spectrum divided by a factor of 5 , representing the UVRR spectrum of the oxy form. To show spectral changes upon ligand dissociation clearly, difference spectra were calculated using the $\mathrm{ClO}_{4}{ }^{-}$ band $\left(934 \mathrm{~cm}^{-1}\right)$ as an internal intensity standard and subtracting the probe-only spectrum from the pump-probe spectrum at each delay time. The probe and pump wavelengths are $233 \mathrm{~nm}$ and $532 \mathrm{~nm}$, respectively. A negative delay time represents the condition that the probe pulse arrived before the pump pulse. Linear background and the Raman bands of water, oxygen gas, glycerol, and the quartz cell have been subtracted. The accumulation time for obtaining each spectrum was $90 \mathrm{~min}$. 

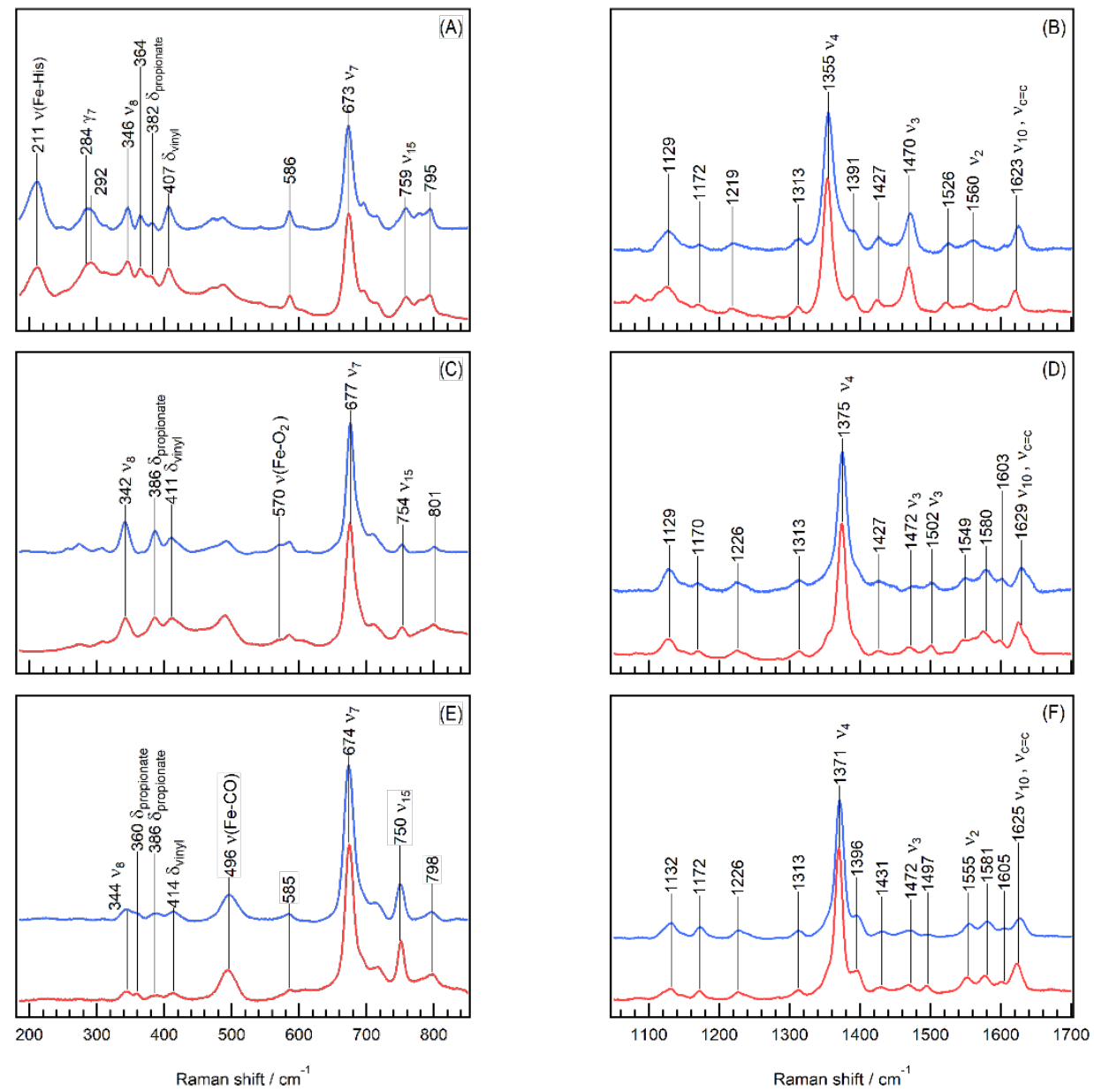

Figure S2. Visible resonance Raman spectra of SmFixL WT and Y496F mutant. (A) and (B), deoxy form; (C) and (D), oxy form; $(\mathrm{E})$ and $(\mathrm{F})$, carbonmonoxy form. Blue and red traces show the spectra of WT and Y496F, respectively. Raman scattering for deoxy form was excited by 441.6-nm line of a He-Cd laser (Kimmon Electric, IK4401R-D) and that for oxy and carbonmonoxy forms was excited by 405-nm line of a diode laser (Innovative Photonics Solutions, I0405SD0045B). The laser power was set to $0.5 \mathrm{~mW}$. Accumulation time for obtaining each spectrum was $15 \mathrm{~min}$. 